Research Article

\title{
Chaotic Dynamics and Chaos Control in a Fractional-Order Satellite Model and Its Time-Delay Counterpart
}

\author{
Ahmed M. Sayed $\left(D,{ }^{1,2}\right.$ A. E. Matouk $(D),{ }^{3,4}$ Sanjay Kumar $\left(D,{ }^{5}\right.$ Vakkar Ali, ${ }^{4}$ \\ and Lahcene Bachioua ${ }^{6}$ \\ ${ }^{1}$ Department of Civil and Environmental Engineering, College of Engineering, Majmaah University, Al-Majmaah 11952, \\ Saudi Arabia \\ ${ }^{2}$ Department of Civil Engineering, College of Engineering, Assiut University, Assiut 71511, Egypt \\ ${ }^{3}$ Department of Mathematics, College of Science Al-Zulf, Majmaah University, Al-Majmaah 11952, Saudi Arabia \\ ${ }^{4}$ College of Engineering, Majmaah University, Al-Majmaah 11952, Saudi Arabia \\ ${ }^{5}$ Amity School of Engineering and Technology, Amity University Patna, Bihar, India \\ ${ }^{6}$ Department of Basic Sciences, Prep. Year, P.O. Box 2440, University of Hail, Hail, Saudi Arabia
}

Correspondence should be addressed to A. E. Matouk; ae.mohamed@mu.edu.sa

Received 25 February 2021; Revised 3 May 2021; Accepted 13 July 2021; Published 22 July 2021

Academic Editor: Shiping Wen

Copyright ( $) 2021$ Ahmed M. Sayed et al. This is an open access article distributed under the Creative Commons Attribution License, which permits unrestricted use, distribution, and reproduction in any medium, provided the original work is properly cited.

\begin{abstract}
Fractional analysis provides useful tools to describe natural phenomena, and therefore, it is more convenient to describe models of satellites. This work illustrates rich chaotic behaviors that exist in a fractional-order model for satellite with and without timedelay. The proof for existence and uniqueness of the satellite model's solution with and without time-delay is shown. Chaos control is achieved in this system via a simple linear feedback control criterion. Chaotic attractors and chaos control are also found in a time-delay version of the proposed fractional-order satellite system. Various tools based on numerical simulations such as 2D and $3 \mathrm{D}$ attractors and bifurcation diagrams are used to illustrate the variety of rich chaotic dynamics in the satellite models.
\end{abstract}

\section{Introduction}

Fractional analysis has become a basic topic for research since it presents appropriate mathematical tools to explain a wide variety of engineering, physical and biological phenomena, and some interdisciplinary topics such as neural networks [1-9]. During the past century, some fractional differential operators were successfully proposed to describe fractional derivatives such as Caputo's type [10] and Riemann-Liouville's type [11]. The aforementioned operators are defined by integration so they are sorted as nonlocal operators with singular kernels. However, the so called Caputo-Fabrizio operator [12] is nonlocal operator with the nonsingular kernel. As a matter of fact, nonlocal operators are a better candidate to model a variety of rich complex dynamics arising in natural phenomena and provide higher adequacies in describing them.
In the past five decades, applications of chaos theory to natural models have received growing interest. Therefore, chaotic dynamics were reported from natural phenomena arising from ecological, economical, physical, and engineering models [13-16]. Moreover, chaotic dynamics in engineering models involving fractional derivatives were reported in [17-20].

Obviously, these kinds of the aforementioned studies help us to understand, quantify, and predict the complex dynamics arising from real world phenomena.

Lyapunov analysis for the stability of the nonlinear system has also become a focal topic for research in integerorder dynamical systems [21-23]. Recently, researchers have developed fractional versions of the Lyapunov stability theory (LST) [24]. Furthermore, the LST has been developed for the case of time-delayed fractional-order systems [25-28]. 
Studying models of satellites has received increasing attention due to their potential applications to scientific, military, and civil activities and space technology [29-33]. On the contrary, chaotic dynamics arising from satellite models have been reported by many researchers [34-38]. In [39], Khan and Kumar reported that chaotic dynamics from a mathematical model of satellite consists of the 3D integerorder system. In addition, Khan and Kumar investigated the chaotic integer-order satellite (IOS) system based on T-S fuzzy modeling [40]. Moreover, in [41], Khan and Kumar reported some conditions for chaos synchronization in the IOS system with time delay. Furthermore, a fractional-order version of the IOS system, namely, the fractional-order satellite (FOS) model, was studied by Kumar et al. [18].

Motivated by the aforementioned statements, we further investigate the chaotic dynamics in the FOS model with and without time delay. We also prove existence and uniqueness of solutions in this model with and without time delay. Then, we intend to suppress chaotic dynamics to the FOS's origin steady state using a simple linear control criterion which depends on a specific selection of the feedback control gains (FCGs). Chaotic attractors and other interesting dynamics are found in a time-delay version of the FOS model. Finally, the abovementioned linear feedback controller is also implemented into a controlled time-delay version of the FOS model. The obtained results show that the time-delay version of the FOS model is also stabilized to its origin equilibrium state when using the same FCGs, whose selection is based on the LST of fractional-order systems with time delay. The numerical results point out that the state variables of the controlled FOS equations are controlled to the origin state faster than its timedelay counterpart does when using the same linear controllers.

\section{Fractional Calculus}

Throughout this work, the following Caputo's type [10] represents the imposed fractional differential operator:

$$
D^{\alpha} \theta(x)=J^{s-\alpha} \theta^{(s)}(x), \quad \alpha \in R^{+},
$$

where $\alpha \in(0,1]$ is the fractional order, namely, the fractional parameter, $\theta^{(s)}$ represents the $s$-order derivative of $\theta(x)$, and $s$ refers to the minimum integer that is not less than the fractional parameter, and $J^{\mu}$ is described by

$$
J^{\mu} \vartheta(x)=\frac{\int_{0}^{x}(x-\chi)^{\mu-1} \vartheta(\chi) \mathrm{d} \chi}{\Gamma(\mu)}, \quad \mu \in R^{+},
$$

where $\Gamma($.$) stands for the Gamma function.$

Then, we take into account the following initial value problem (IVP):

$$
\begin{aligned}
& D^{\alpha} \xi(t)=\theta(t, \xi(t)), \quad 0 \leq t \leq T, \\
& \xi^{(m)}(0)=\xi_{0}^{(m)}, \quad m=0,1, \ldots, s-1,
\end{aligned}
$$

which is equivalent to the nonlinear Volterra integral equation of the second kind, provided that the function $\theta$ is continuous; then, IVP (3) is solved by

$$
\xi(t)=\sum_{m=0}^{s-1} \frac{t^{m}}{m !} \xi_{0}^{(m)}+\frac{\left[\int_{0}^{t}\left(\theta(\zeta, \xi(\zeta)) /(t-\zeta)^{1-\alpha}\right) \mathrm{d} \zeta\right]}{\Gamma(\alpha)} .
$$

According to Diethelm et al. [42, 43], the predictorcorrectors (PECE) method can be used to discretize equation (4) as follows:

$$
\begin{aligned}
\xi_{h}\left(t_{n+1}\right)= & \sum_{m=0}^{s-1} \frac{t_{n+1}^{m}}{m !} \xi_{0}^{(m)}+\frac{\theta\left(t_{n+1}, \xi_{h}^{P}\left(t_{n+1}\right)\right) h^{\alpha}}{\Gamma(2+\alpha)} \\
& +\frac{\sum_{j=0}^{n} a_{j, n+1} \theta\left(t_{j}, \xi_{n}\left(t_{j}\right)\right) h^{\alpha}}{\Gamma(2+\alpha)},
\end{aligned}
$$

where $h=T / N$ for some integer $N$ defined using the uniform grid $\left\{t_{n}=h n: n=0,1, \ldots, N\right\}, \xi_{h}\left(t_{n}\right)$ refers to an approximation to $\xi\left(t_{n}\right)$, and the coefficient $a_{j, n+1}$ defined in the last term of RHS of equation (5) is described as

$$
a_{j, n+1}= \begin{cases}n^{\alpha+1}-(n-\alpha)(n+1)^{\alpha}, & \text { if } j=0, \\ (n-j+2)^{\alpha+1}-2(n-j+1)^{\alpha+1}+(n-j)^{\alpha+1}, & \text { if } 1 \leq j \leq n, \\ 1, & \text { if } j=n+1,\end{cases}
$$

and the predictor $\xi_{h}^{P}\left(t_{n+1}\right)$ is obtained from

$$
\begin{aligned}
& \xi_{h}^{P}\left(t_{n+1}\right)=\sum_{m=0}^{s-1} \frac{t_{n+1}^{m}}{m !} \xi_{0}^{(m)}+\frac{\sum_{j=0}^{n} b_{j, n+1} \theta\left(t_{j}, \xi_{n}\left(t_{j}\right)\right)}{\Gamma(\alpha)}, \\
& b_{j, n+1}=\frac{\left[(n-j+1)^{\alpha}-(n-j)^{\alpha}\right] h^{\alpha}}{\alpha}, \quad 0 \leq j \leq n .
\end{aligned}
$$

A modification of the PECE scheme was presented in [44] to integrate fractional-order equations with time delay. It can also be described as follows; for the delayed time fractional equation,

$$
D^{\alpha} \xi(t)=\theta(t, \xi(t), \xi(t-\tau)), \quad 0 \leq t \leq T, \xi(t)=\phi(t) \text {, and }-\tau \leq t \leq 0,
$$

where $\phi(t)$ can be denoted by an element of the Banach space of all continuous functions over $[-\tau, 0]$, that is, $C\left([-\tau, 0], R^{n}\right)$. Then, assume that

$$
\xi_{h}\left(t_{j}\right)=\phi\left(t_{j}\right), \quad j=-m, 1-m, \ldots,-1,0,
$$

where $\tau=m h$ and $h=T / N$ for some integer $N$ defined using the uniform grid $\left\{t_{n}=h n: n=-m, 1-m, \ldots,-1,0,1\right.$, $\ldots, N\}$ with the following notation:

$$
\xi_{h}\left(t_{j}-\tau\right)=\xi_{h}(h(j-m))=\xi_{h}\left(t_{j-m}\right), \quad j=0,1, \ldots, N .
$$

So, $\xi_{h}\left(t_{n+1}\right)$ can be calculated via

$$
\xi\left(t_{n+1}\right)=\varphi(0)+\frac{\left[\int_{0}^{t_{n+1}}\left(\theta(\zeta, \xi(\zeta), \xi(\zeta-\tau)) /\left(t_{n+1}-\zeta\right)^{1-\alpha}\right) d \zeta\right]}{\Gamma(\alpha)} .
$$


Now, according to [44], the formula for the corrector variable is obtained via

$$
\begin{aligned}
\xi\left(t_{n+1}\right)= & \phi(0)+\frac{\theta\left(t_{n+1}, \xi_{h}\left(t_{n+1}\right), \xi_{h}\left(t_{n+1-m}\right)\right) h^{\alpha}}{\Gamma(2+\alpha)} \\
& +\frac{\sum_{j=0}^{n} a_{j, n+1} \theta\left(t_{j}, \xi_{h}\left(t_{j}\right), \xi_{h}\left(t_{j-m}\right)\right) h^{\alpha}}{\Gamma(2+\alpha)},
\end{aligned}
$$

and the predictor variable can be evaluated via

$$
\xi_{h}^{P}\left(t_{n+1}\right)=\phi(0)+\frac{\sum_{j=0}^{n} b_{j, n+1} \theta\left(t_{j}, \xi_{h}\left(t_{j}\right), \xi_{h}\left(t_{j-m}\right)\right)}{\Gamma(\alpha)} .
$$

Also, we introduce the following basic lemmas.

Lemma 1 (see [45]). Assume that the vector-value function $\xi(t) \in R^{n}$ is continuous and differentiable. Then, $\forall t \geq t_{0}$, and the following inequality holds:

$$
0.5 D^{\alpha} \xi^{T} \xi \leq \xi^{T} D^{\alpha} \xi .
$$

Lemma 2 (see [46]). Assume that a nonnegative function $P(t) \in R$ is given that it is continuously differentiable and satisfies the following conditions (as $\alpha \in(0,1)$ ):

$D^{\alpha} P(t) \leq-\eta_{1} P(t)+\eta_{2} P(t-\tau), \quad P(t)=\Psi(t) \geq 0$ and $-\tau \leq t \leq 0$, where $t$ is a nonnegative real number and $\eta_{i} \in R^{+}$, $i=1$ and 2. If $\eta_{1}>\eta_{2} \forall \tau \in R^{+}$and $\forall \Psi(t) \geq 0$, then

$$
\lim _{t \longrightarrow+\infty} P(t)=0 \text {. }
$$

Remark 1. A function $\theta(t, \xi(t), \xi(t-\tau)) \in C\left([0, T], R^{n}\right)$ is called a Lipschitz continuous if $\exists \eta>0$ such that the constant $\eta$ satisfies the following inequality:

$$
\begin{aligned}
& \left\|\theta(t, \xi(t), \xi(t-\tau))-\theta\left(t, \xi^{\prime}(t), \xi^{\prime}(t-\tau)\right)\right\| \\
& \leq \eta\left(\left\|\xi(t)-\xi^{\prime}(t)\right\|+\left\|\xi(t-\tau)-\xi^{\prime}(t-\tau)\right\|\right),
\end{aligned}
$$

for any $t \in[0, T]$, where $\|$.$\| refers to a matrix norm.$

If the delayed time fractional system has the form

$$
\begin{aligned}
D^{\alpha} \xi(t) & =M_{0} \xi(t)+M_{1} \xi(t-\tau)+\theta(t, \xi(t), \xi(t-\tau)), \quad 0 \leq t \leq T, \\
\xi(t) & =\phi(t), \quad-\tau \leq t \leq 0,
\end{aligned}
$$

where $T \in R^{+}$or $T$ represents $+\infty, \quad \theta(t, \xi(t), \xi(t-\tau))$ $\in C\left([0, T], R^{n}\right), \tau>0$, and $M_{0}$ and $M_{1}$ are constant matrices, then we obtain the following results:

Remark 2 (see [47]). A necessary and sufficient condition for a continuously differentiable function $\xi:[-\tau, T] \longrightarrow R^{n}$ to be a solution of (18) is

$$
\xi(t)= \begin{cases}\phi(0)+\frac{\left[\int_{0}^{t}\left(M_{0} \xi(\zeta)+M_{1} \xi(\zeta-\tau)+\theta(\zeta, \xi(\zeta), \xi(\zeta-\tau)) /(t-\zeta)^{1-\alpha}\right) \mathrm{d} \zeta\right]}{\Gamma(\alpha)}, & \text { as } t \in[0, T], \\ \phi(t), & \text { as } t \in[-\tau, 0] .\end{cases}
$$

Theorem 1 (see [47]). If the nonlinear function $\theta(t, \xi(t)$, $\xi(t-\tau)) \in C\left([0, T], R^{n}\right)$ is Lipschitz continuous, then there exists a unique and continuous solution for general system (18).

Finally, we end this section with the following lemmas:

Lemma 3 (see [48]). Consider that the generalized fractional system $D^{\alpha} \xi(t)=\theta(\xi), \alpha \in(0,1]$, such that there exists a Lyapunov function $P(\xi)$ for the equilibrium solution $\bar{\xi}$ in case of $\alpha=1$; then, $\bar{\xi}$ is at least locally asymptotically stable (LAS) as $\alpha \in(0,1)$.

Lemma 4 (see [48]). Let $M \in C^{n \times n}$ be a Hermitian matrix whose all principal minor determinants $P_{1}, P_{2}, \ldots, P_{n} \in R^{+}$; then, the quantity $-\xi^{T} M \xi \in R^{-}$and $\forall \xi \neq 0$ belong to a domain $\Omega \subset C^{n}$ that contains a neighborhood of $\xi=0$.

\section{The Chaotic FOS Model}

The FOS is described by Kumar et al. [18]:

$$
\begin{aligned}
& D^{\alpha} x(t)=\frac{1}{3} y z-a x+\frac{1}{\sqrt{6}} z, \\
& D^{\alpha} y(t)=-x z+b y, \\
& D^{\alpha} z(t)=x y-\sqrt{6} x-c z,
\end{aligned}
$$

where $a, b$, and $c \in R^{+}$. FOS model (20) exhibits a variety of rich dynamics including chaotic attractors, as outlined in Figure 1, using $a=0.4, b=0.175$, and $c=0.4$. The corresponding bifurcation diagrams are depicted in Figure 2. 


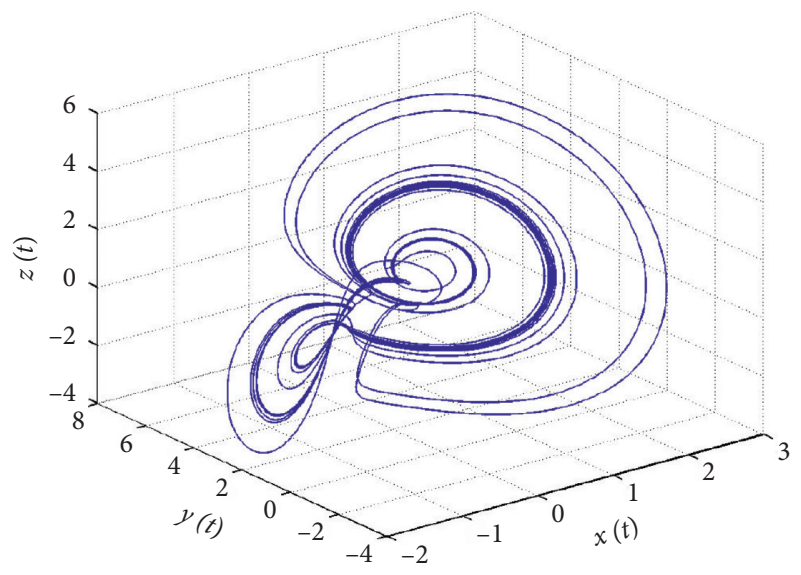

(a)

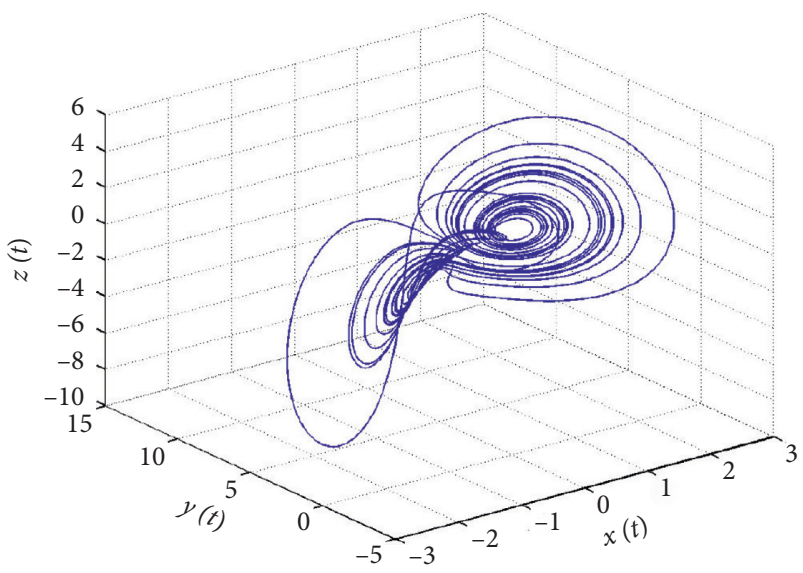

(c)

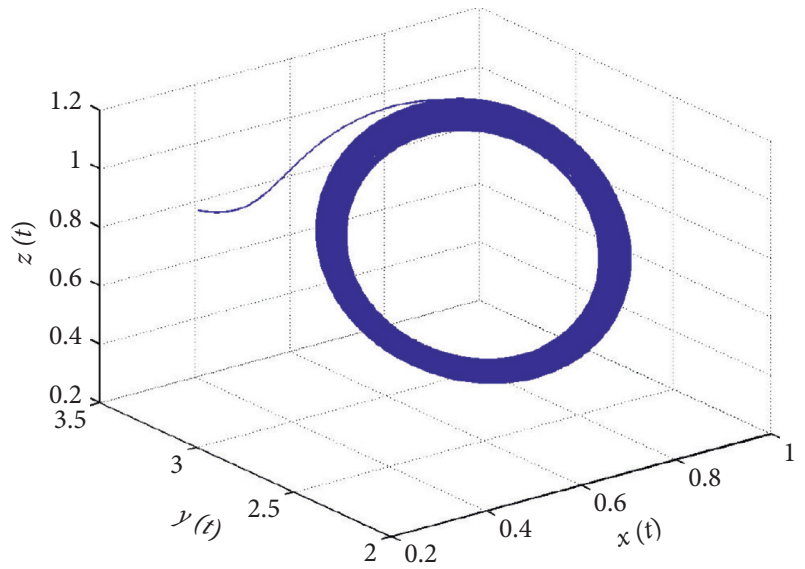

(e)

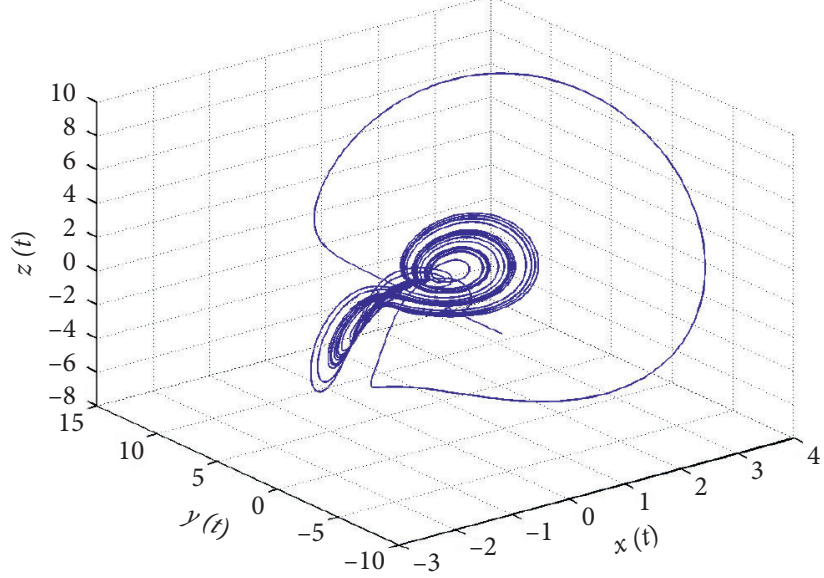

(b)

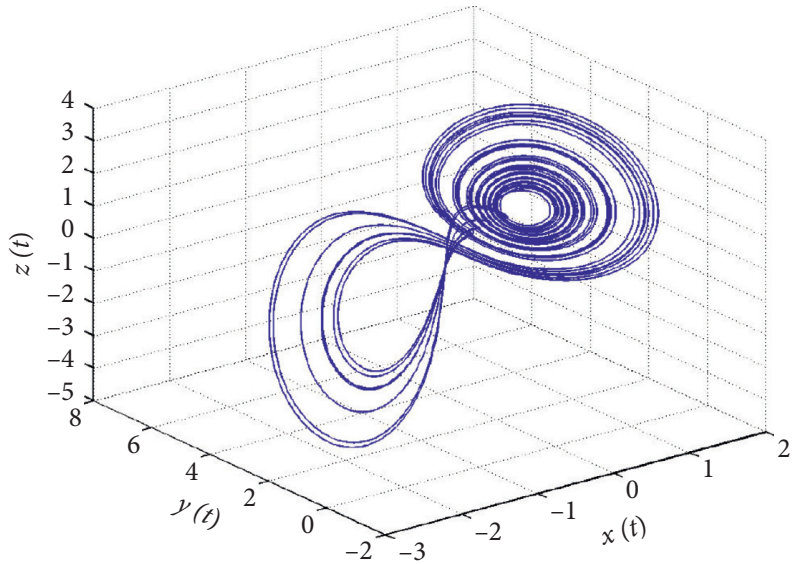

(d)

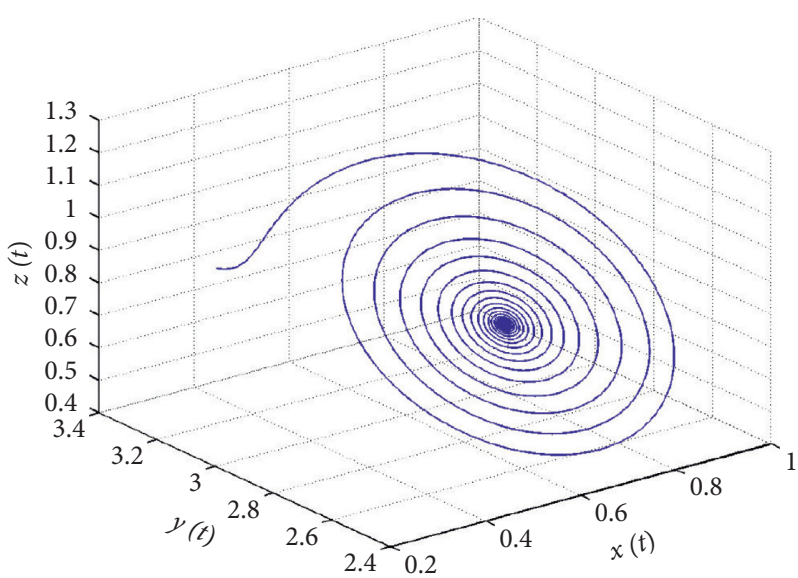

(f)

FIGURE 1: 3D plots of FOS model (20) with $a=0.4, b=0.175$, and $c=0.4$ showing that (a) chaos exists at $\alpha=0.99$; (b) chaos exists at $\alpha=0.97$; (c) chaos exists at $\alpha=0.95$; (d) chaos exists at $\alpha=0.93$; (e) quasi-periodic attractor exists at $\alpha=0.92$; (f) asymptotic attractor exists at $\alpha=0.90$.

Calculations of the Lyapunov spectrum show that the FOS system is still chaotic for a wide range of parameters beyond $\alpha=0.9$ since the maximal Lyapunov exponents are positive. This interesting observation is depicted in Figure 3, and it verifies the existence of rich chaotic dynamics in the FOS system.

\section{Solution's Existence and Uniqueness in the FOS Model}

The FOS system is represented in the following form:

$$
D^{\alpha} U(t)=G(U(t)), \quad t \in(0, T], U(0)=U_{0},
$$




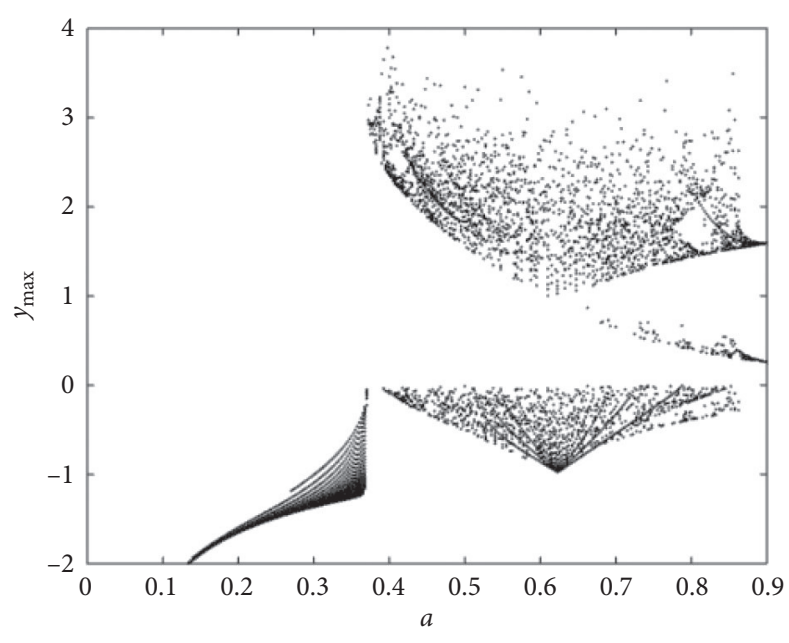

(a)

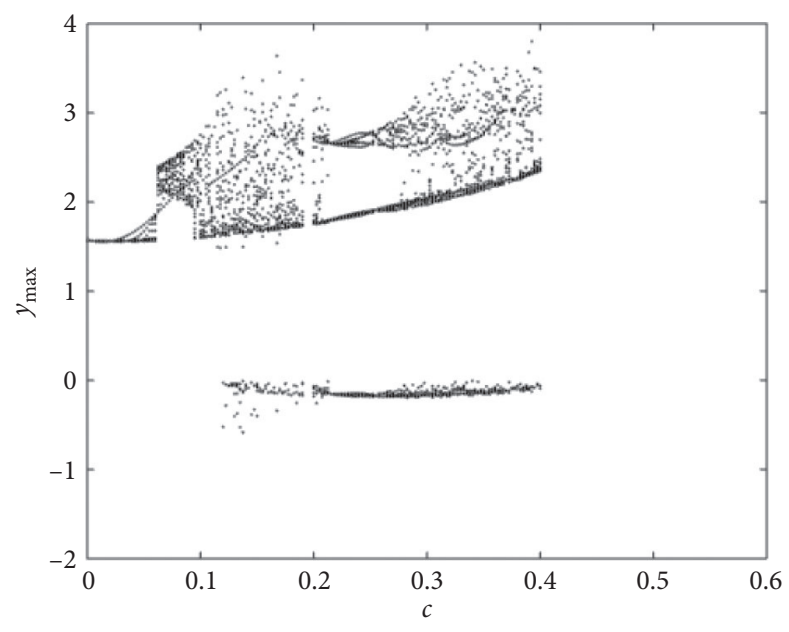

(c)

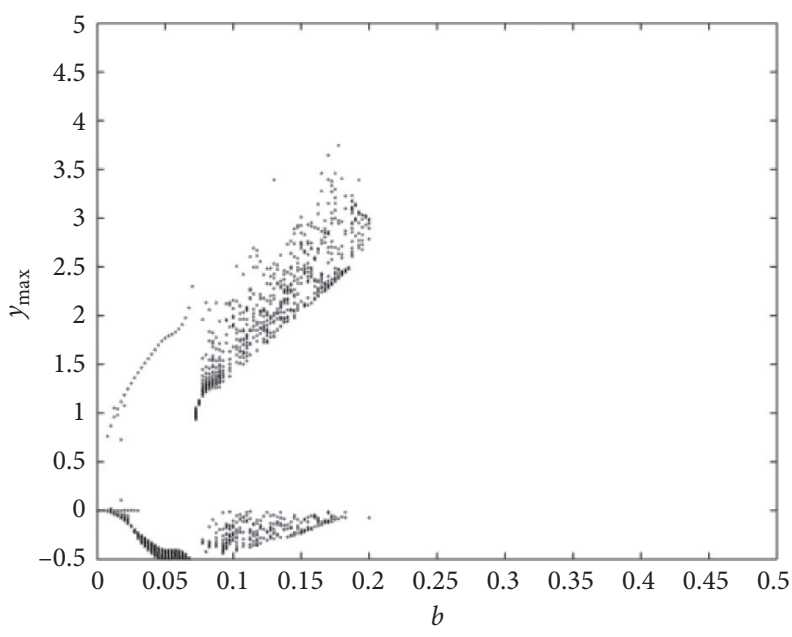

(b)

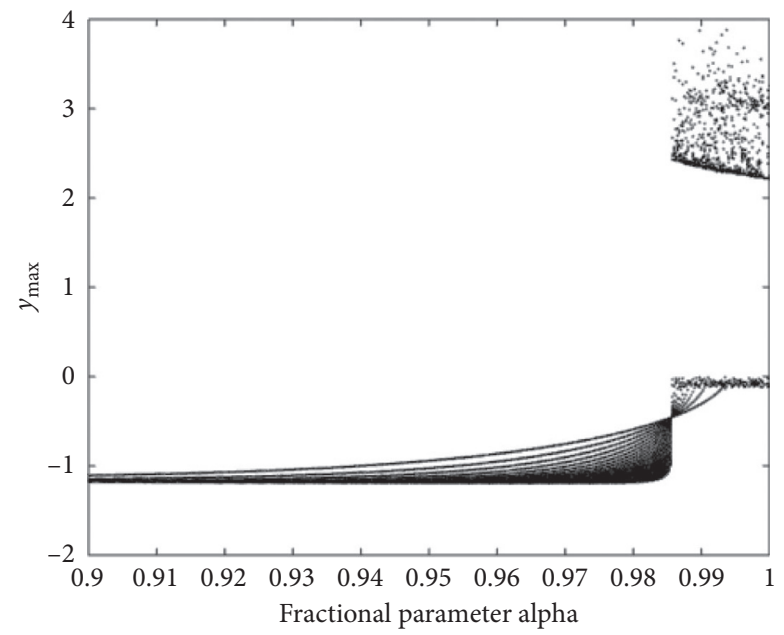

(d)

FIgUre 2: Bifurcation diagram of FOS model (20) as varying (a) $a$ with $b=0.175, c=0.4$, and $\alpha=0.99$; (b) $b$ with setting $a=0.4, c=0.4$, and $\alpha=0.99$; (c) $c$ with setting $b=0.175, a=0.4$, and $\alpha=0.99$; (d) $\alpha$ with setting $a=0.4, b=0.175$, and $c=0.4$.

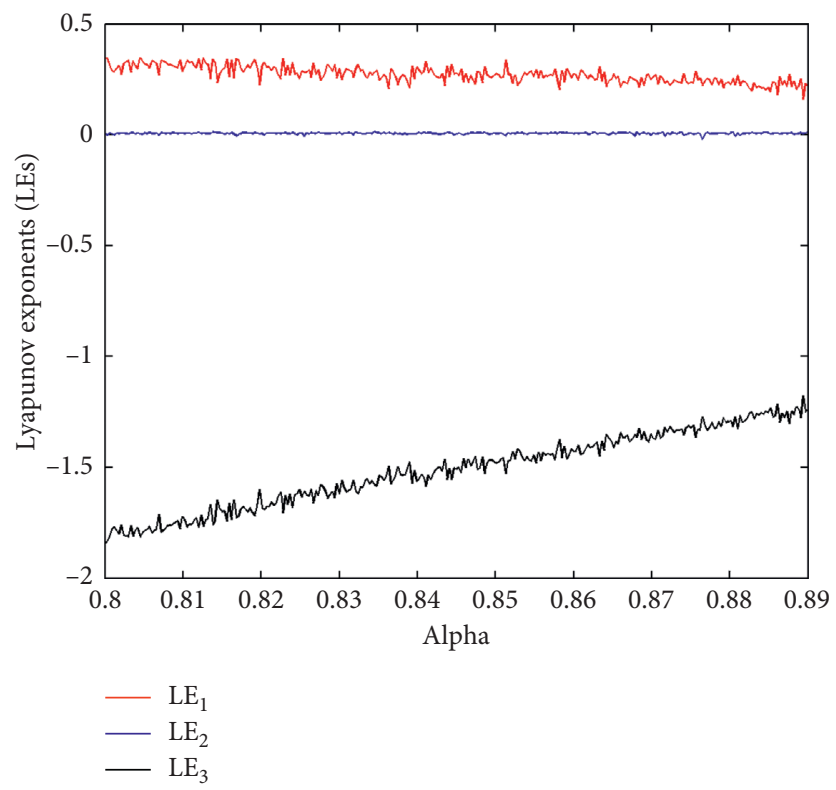

FIGURE 3: Lyapunov spectrum of FOS model (20) as the parameter $\alpha \in[0.8,0.9)$ with setting $a=0.4, b=0.175$, and $c=0.4$. 
where

$$
\begin{aligned}
U & =\left[\begin{array}{l}
x \\
y \\
z
\end{array}\right], \\
U_{0} & =\left[\begin{array}{l}
x_{0} \\
y_{0} \\
z_{0}
\end{array}\right], \\
G(U) & =\left[\begin{array}{c}
\frac{1}{3} y z-a x+\frac{1}{\sqrt{6}} z \\
-x z+b y \\
x y-\sqrt{6} x-c z
\end{array}\right] .
\end{aligned}
$$

Let $C[0, T]$ be a class of continuous functions. Hence, the supremum norm is defined as

$$
\|\Omega\|=\sup _{t \in(0, T]}|\Omega(t)|, \quad \Omega(t) \in C[0, T] .
$$

Furthermore, for a matrix $A=\left[a_{i j}[t]\right]$, the norm is described as

$$
\|A\|=\sum_{i, j} \sup _{t \in(0, T]}\left|a_{i j}[t]\right| .
$$

We next discuss the existence and uniqueness of the solution of the FOS model in the region $\Phi \times(0, T]$ where

$$
\Phi=\{(x, y, z): \max (|x|,|y|,|z|) \leq v\} .
$$

According to Remark 2, with setting $\tau=0$, the solution of chaotic FOS system (20) is obtained as

$$
U=U_{0}+\frac{1}{\Gamma(\alpha)} \int_{0}^{t}(t-\chi)^{\alpha-1} G(U(\chi)) \mathrm{d} \chi=\Psi(U) .
$$

So,

$\Psi\left(U_{1}\right)-\Psi\left(U_{2}\right)=\frac{1}{\Gamma(\alpha)} \int_{0}^{t}(t-\chi)^{\alpha-1}\left[G\left(U_{1}(\chi)\right)-G\left(U_{2}(\chi)\right)\right] \mathrm{d} \chi$.

Consequently, we obtain

$\left|\Psi\left(U_{1}\right)-\Psi\left(U_{2}\right)\right| \leq \int_{0}^{t}\left|\frac{(t-\chi)^{\alpha-1}}{\Gamma(\alpha)}\left[G\left(U_{1}(\chi)\right)-G\left(U_{2}(\chi)\right)\right]\right| \mathrm{d} \chi$,

which is reduced to

$$
\begin{aligned}
\left\|\Psi\left(U_{1}\right)-\Psi\left(U_{2}\right)\right\| \leq & \frac{T^{\alpha}}{\Gamma(\alpha+1)} \max \\
& \left\{\sqrt{6}+a+2 v, \frac{\sqrt{6}}{6}+c+\frac{4}{3} v, b+\frac{4}{3} v\right\} \\
\left\|U_{1}-U_{2}\right\|, \leq \eta\left\|U_{1}-U_{2}\right\|, &
\end{aligned}
$$

where

$$
\eta=\frac{T^{\alpha}}{\Gamma(\alpha+1)} \max \left\{\sqrt{6}+a+2 v, \frac{\sqrt{6}}{6}+c+\frac{4}{3} v, b+\frac{4}{3} v\right\} .
$$

If $\eta<1$, then $U=\Psi(U)$ is a contraction mapping, and the following theorem gives a sufficient condition for existence and uniqueness of the solution of FOS model (20).

Theorem 2. A sufficient condition for solution's existence and uniqueness of FOS system (20) in the region $\Phi \times(0, T]$ with initial conditions $U(0)=U_{0}$ and $t \in(0, T]$ is

$$
\eta=\frac{T^{\alpha}}{\Gamma(\alpha+1)} \max \left\{\sqrt{6}+a+2 v, \frac{\sqrt{6}}{6}+c+\frac{4}{3} v, b+\frac{4}{3} v\right\} .
$$

\section{Chaos Control of the Chaotic FOS Model via a Linear Feedback Criterion}

Suppose that $X=\left(x^{e}, y^{e}, z^{e}\right)$ denotes an equilibrium point of chaotic FOS model (20) and $k_{1}, k_{2}$, and $k_{3}$ are the positive feedback control gains (FCGs). Then, a controlled form of FOS model (20) is introduced by

$$
\begin{aligned}
& D^{\alpha} x(t)=\frac{1}{3} y z-a x+\frac{1}{\sqrt{6}} z-k_{1}\left(x-x^{e}\right), \\
& D^{\alpha} y(t)=-x z+b y-k_{2}\left(y-y^{e}\right), \\
& D^{\alpha} z(t)=x y-\sqrt{6} x-c z-k_{3}\left(z-z^{e}\right) .
\end{aligned}
$$

In case of the node equilibrium point of chaotic FOS model (20), we have the following theorem:

Theorem 3. The chaotic behaviors in FOS model (20) are suppressed to its zero steady state if the following conditions hold:

$$
\begin{aligned}
& k_{1}>-a, \\
& k_{2}>b, \\
& k_{3}>-c-\frac{1}{144\left(a+k_{1}\right)}\left(\frac{5 \sqrt{6}}{6}+2 \mu_{y}\right)^{2},
\end{aligned}
$$

where $\mu_{y}>|y|$.

Proof. Chaotic FOS system (32) matches the following Lyapunov function:

$$
V(x, y, z)=0.5\left(x^{2}+y^{2}+z^{2}\right) .
$$

Hence, we obtain 


$$
\begin{aligned}
D^{1} V(x, y, z)= & x(t) D^{1} x(t)+y(t) D^{1} y(t)+z(t) D^{1} z(t) \\
= & -\left(a+k_{1}\right) x^{2}+\left(b-k_{2}\right) y^{2}-\left(c+k_{3}\right) z^{2} \\
& -\frac{5 \sqrt{6}}{6} x z+\frac{1}{3} x y z \\
\leq & -\left(a+k_{1}\right)|x|^{2}+\left(b-k_{2}\right)|y|^{2}-\left(c+k_{3}\right)|z|^{2} \\
& +\frac{1}{6}\left(\frac{5 \sqrt{6}}{6}+2 \mu_{y}\right)|x||z|=-\mathrm{X}^{T} \mathrm{QX},
\end{aligned}
$$

where

$$
\begin{aligned}
\mathrm{X} & =\left[\begin{array}{ccc}
|x||y||z| & ]^{T}, \\
a+k_{1} & 0 & -\left(\frac{5 \sqrt{6}}{72}+\frac{1}{6} \mu_{y}\right) \\
0 & k_{2}-b & 0 \\
-\left(\frac{5 \sqrt{6}}{72}+\frac{1}{6} \mu_{y}\right) & 0 & c+k_{3}
\end{array}\right] .
\end{aligned}
$$

The Hermitian matrix $Q$ is strictly positive if all conditions (33) hold. Therefore, according to Lemma 4, it follows that the integer-order derivative of $V$ is negative when $(x, y, z)$ is not origin, while it is vanished at the origin. Then, based on Lemma 3, the origin equilibrium of chaotic FOS model (20) is at least LAS.

Controlled FOS model (32) is numerically integrated with $a=0.4, b=0.175, c=0.4$, and $\alpha=0.96$. Now, according to inequalities (33), the FCGs can be chosen as $k_{1}=1, k_{2}=$ 2 , and $k_{3}=1$. The results are given in Figure 4 .

\section{Chaotic Dynamics in the FOS Delay Model}

A time-delay version of FOS model (20) is represented here by

$$
\begin{aligned}
& D^{\alpha} x(t)=\frac{1}{3} y(t) z(t)-a x(t)+\frac{1}{\sqrt{6}} z(t), \\
& D^{\alpha} y(t)=-x(t) z(t)+b y(t-\tau), \\
& D^{\alpha} z(t)=x(t) y(t)-\sqrt{6} x(t)-c z(t),
\end{aligned}
$$

where $\tau$ is the delay constant. Chaotic attractors in FOS delay model (37) are depicted in Figure 5 using different values of $\alpha$ and fixing other model's parameters at $a=0.4, b=0.175, c=0.4$, and $\tau=0.2$. Moreover, chaotic behaviors are depicted in $x(t) x(t-\tau)$ plane, as shown in Figure 6. Also, the bifurcation plot corresponding to the aforementioned parameter set is illustrated in Figure 7.

To prove existence and uniqueness of FOS delay model (37), we carry out similar analysis, as given by Theorem 2 , that lead to the following inequality (for any $t \in[0, T]$ ):

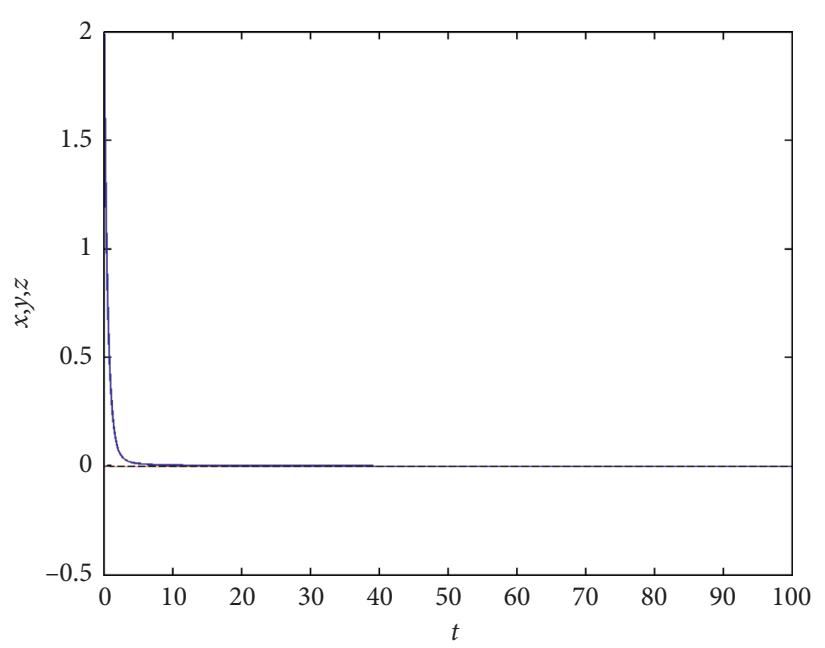

$-x$
$-\quad y$
$---z$

Figure 4: Trajectories of chaotic FOS system (32) converge to the origin steady state using $a=0.4, b=0.175, c=0.4$, and $\alpha=0.96$ and the FCGs $k_{1}=1, k_{2}=2$, and $k_{3}=1$.

$$
\begin{aligned}
& \left\|\Psi\left(U_{1}(t), U_{1}(t-\tau)\right)-\Psi\left(U_{2}(t), U_{2}(t-\tau)\right)\right\| \\
& \leq \eta\left(\left\|U_{1}(t)-U_{2}(t)\right\|+\left\|U_{1}(t-\tau)-U_{2}(t-\tau)\right\|\right),
\end{aligned}
$$

where

$$
\eta=\frac{T^{\alpha}}{\alpha \Gamma(\alpha)} \max \left\{\frac{1}{\sqrt{6}}+\frac{4}{3} v+c, b, 2 v+\sqrt{6}+a\right\}>0 .
$$

Thus, $\Psi(t, U(t), U(t-\tau)) \in C\left([0, T], R^{n}\right)$ has been shown to be Lipschitz continuous. Then, according to Theorem 1, there exists a unique and continuous solution for FOS delay model (37).

\section{Chaos Control of the Chaotic FOS Delay Model via a Linear Feedback Criterion}

A controlled version of FOS delay model (37) to its origin steady state is described as

$$
\begin{aligned}
& D^{\alpha} x(t)=\frac{1}{3} y(t) z(t)-a x(t)+\frac{1}{\sqrt{6}} z(t)-k_{1} x(t), \\
& D^{\alpha} y(t)=-x(t) z(t)+b y(t-\tau)-k_{2} y(t) \\
& D^{\alpha} z(t)=x(t) y(t)-\sqrt{6} x(t)-c z(t)-k_{3} z(t)
\end{aligned}
$$

Theorem 4. The chaotic behaviors in FOS delay model (40) are suppressed to their zero steady state if all the FCGs satisfy the inequality $\eta>b / 4$, where $\eta=\min \left\{a+k_{1}-\gamma, k_{2}-(b / 2)\right.$, $\left.c+k_{3}-\gamma\right\}$ and $\gamma=(1 / 12)\left((5 \sqrt{6} / 6)+2 \mu_{y}\right)$.

Proof. The following Lyapunov function is constructed for controlled FOS delay system (40): 


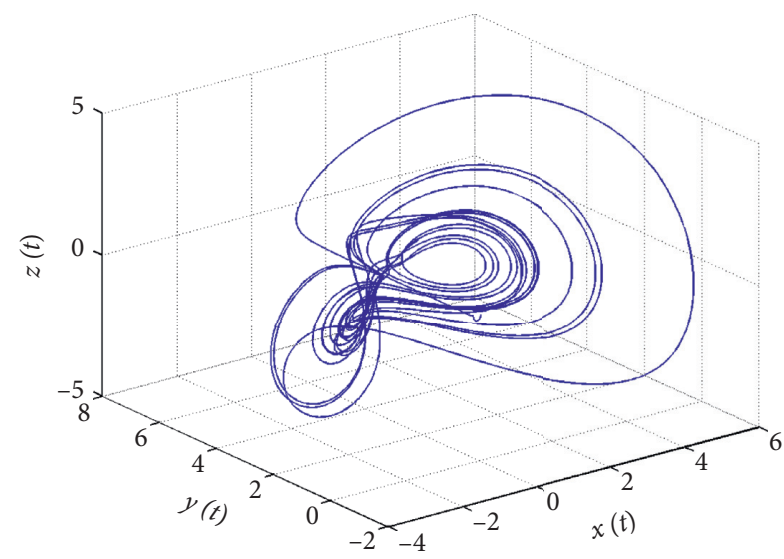

(a)

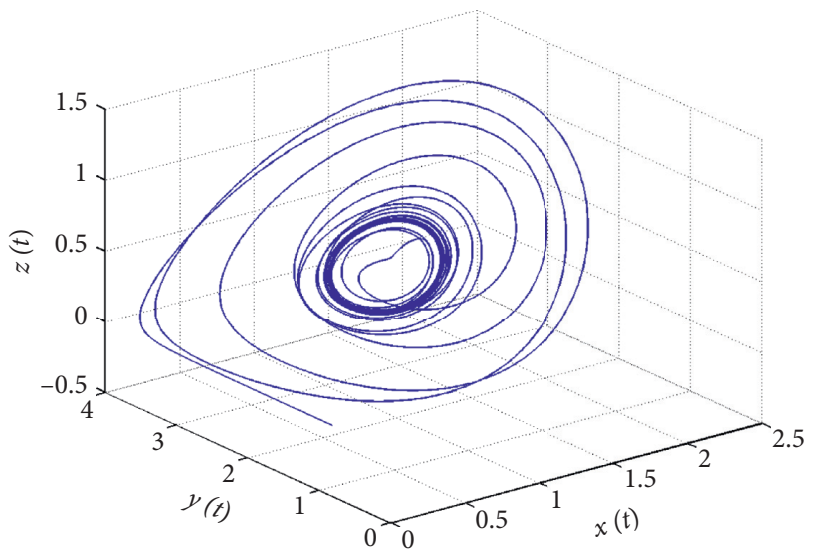

(c)

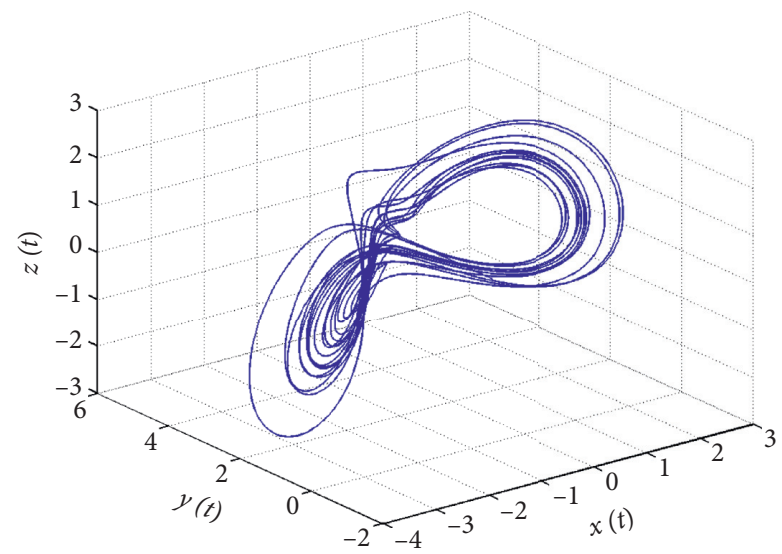

(b)

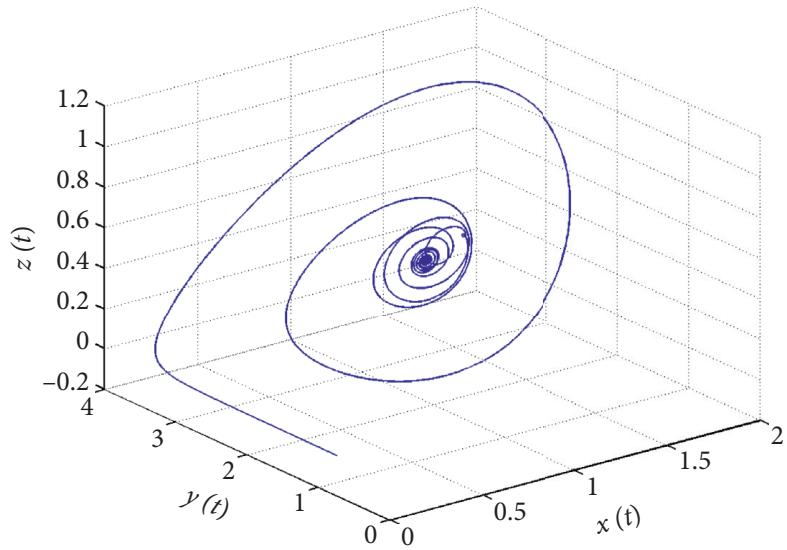

(d)

FIgURE 5: 3D plots of FOS model (37) with $a=0.4, b=0.175, c=0.4$, and $\tau=0.2$, showing that (a) chaos exists at $\alpha=1$; (b) chaos exists at $\alpha=0.99$; (c) quasi-periodic attractor exists at $\alpha=0.95$; (d) asymptotic attractor exists at $\alpha=0.90$.

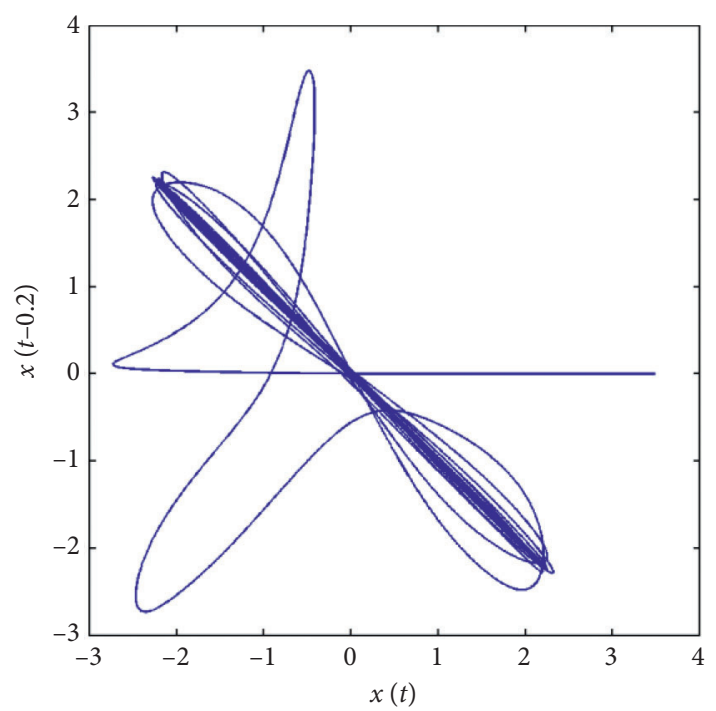

Figure 6: Chaotic solution of FOS delay model (37) in $x(t) x(t-\tau)$ plane with $a=0.4, b=0.175, c=0.4, \alpha=0.995$, and $\tau=0.2$.

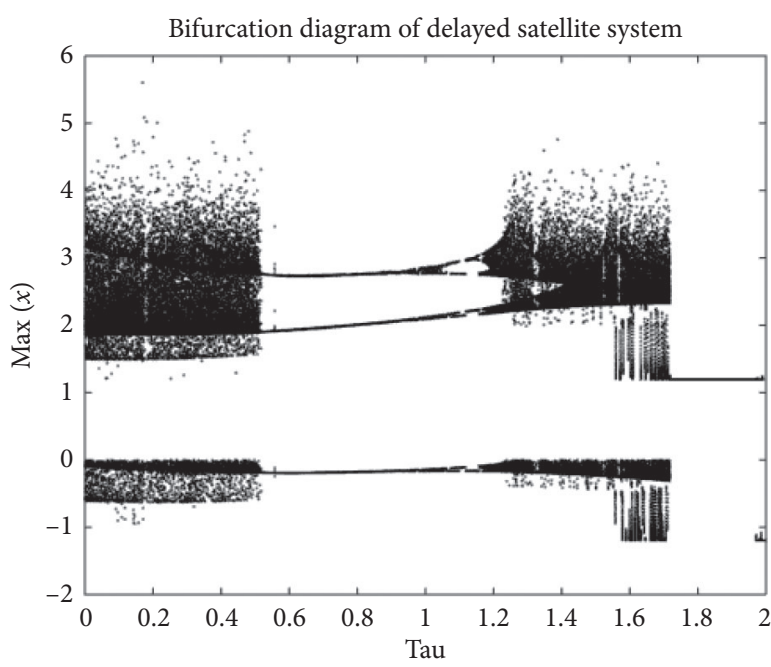

FIGURE 7: Bifurcation diagram of FOS delay model (37) with $a=$ $0.4, b=0.175, c=0.4$, and $\alpha=1$ and varying $\tau$. 


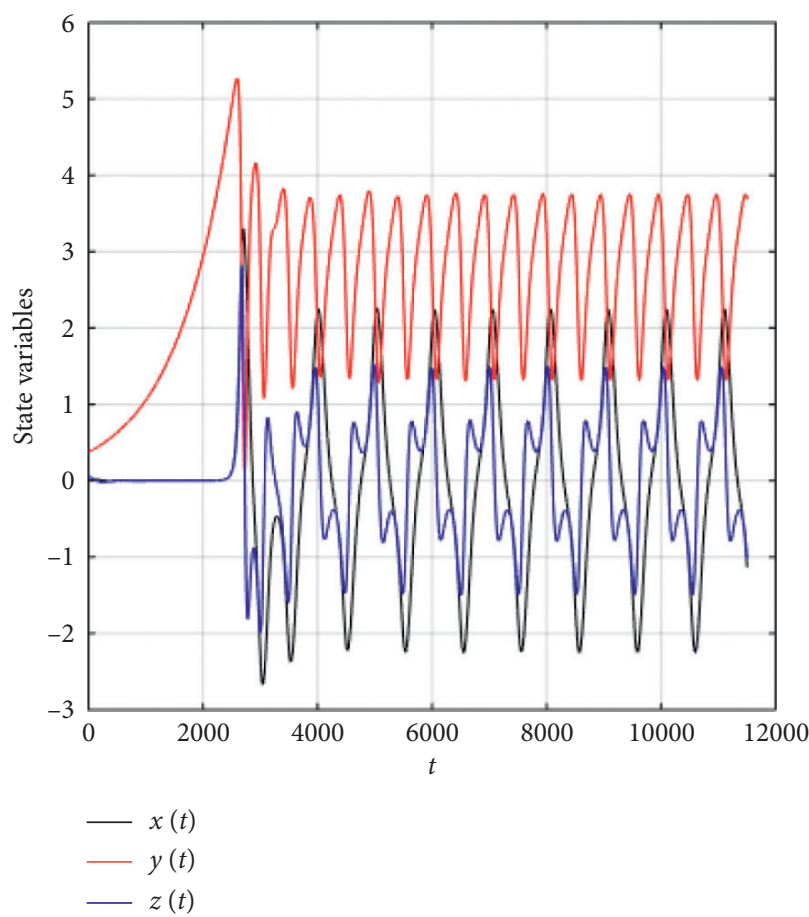

(a)

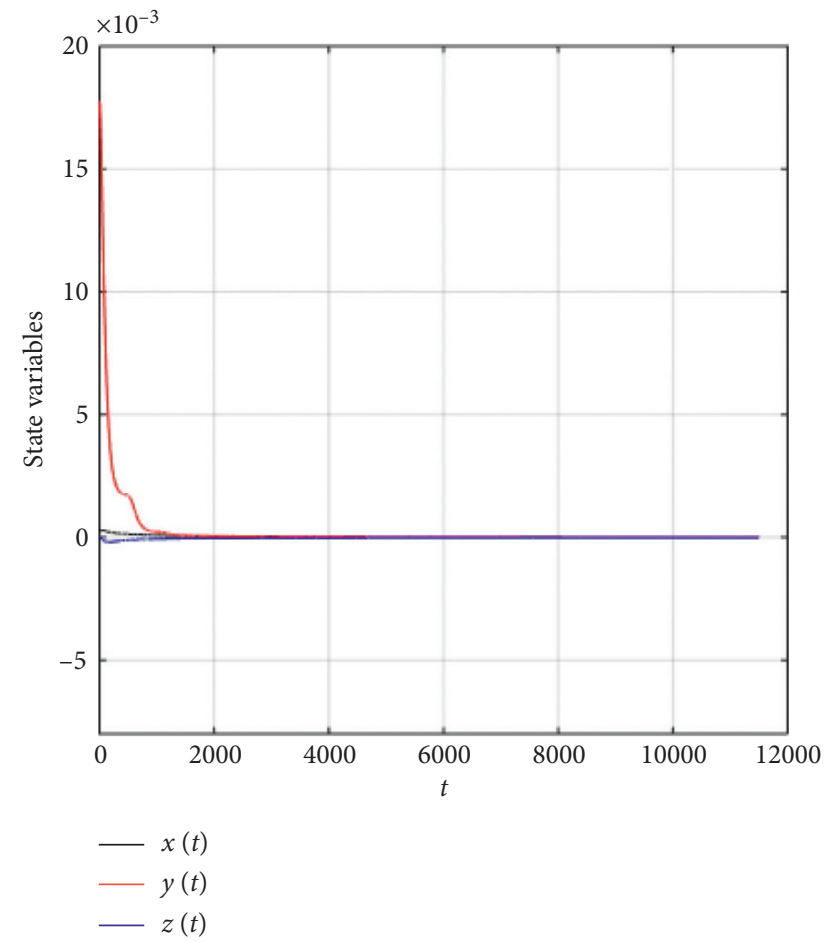

(b)

FIgURE 8: Trajectories of chaotic FOS delay system (40) with $a=0.4, b=0.175, c=0.4$, and $\alpha=0.99$ (a) are not stabilized to $(0,0,0)$ when no control is applied and (b) converge to the origin steady state using the FCGs $k_{1}=1, k_{2}=2$, and $k_{3}=1$.

$$
P(t)=0.5\left(x^{2}+y^{2}+z^{2}\right)
$$

Hence, according to Lemma 1, we obtain

$$
\begin{aligned}
D^{\alpha} P(t) \leq & x(t) D^{\alpha} x(t)+y(t) D^{\alpha} y(t)+z(t) D^{\alpha} z(t) \\
= & -\left(a+k_{1}\right)|x(t)|^{2}-k_{2}|y(t)|^{2}-\left(c+k_{3}\right)|z(t)|^{2} \\
& +b|y(t)||y(t-\tau)|+2 \gamma|x(t)||z(t)| \\
\leq & -\left(a+k_{1}\right)|x(t)|^{2}-k_{2}|y(t)|^{2}-\left(c+k_{3}\right)|z(t)|^{2} \\
& +\frac{b}{2}\left(|y(t)|^{2}+|y(t-\tau)|^{2}\right)+\gamma\left(|x(t)|^{2}+|z(t)|^{2}\right) \\
= & -\left(a+k_{1}-\gamma\right)|x(t)|^{2}-\left(k_{2}-\frac{b}{2}\right)|y(t)|^{2} \\
& -\left(c+k_{3}-\gamma\right)|z(t)|^{2}+\frac{b}{2}|y(t-\tau)|^{2} \\
\leq & -2 \eta P(t)+\frac{b}{2} P(t-\tau) .
\end{aligned}
$$

Referring to Lemma 2, the controlled system is stabilized to the origin steady state if we choose $\eta>b / 4$.

Controlled FOS model (40) is integrated with $a=0.4, b=0.175, c=0.4$, and $\alpha=0.99$. Now, according to
Theorem 4, the FCGs can be chosen as $k_{1}=1, k_{2}=2$, and $k_{3}=1$. The results are given in Figure 8 .

\section{Conclusion}

This work has investigated the rich chaotic behaviors that existed in the FOS model with and without time delay. Various numerical techniques such as three-dimensional attractors, bifurcation diagrams, and Lyapunov spectrum have been employed to examine the existence of chaotic dynamics in the FOS model. We also proved the solution's existence and uniqueness in the FOS equations with and without time delay. Furthermore, a simple linear control criterion was successfully applied to achieve chaos control in the FOS model. This method is very simple and easy to be implemented in the model.

On the contrary, a rich variety of chaotic dynamics in a time-delayed version of the FOS model has been shown using various numerical experiments such as two- and threedimensional attractors and bifurcation diagrams. Finally, the abovementioned linear feedback controller has also been implemented into the FOS model with time delay. The obtained results show that the time-delay version of the FOS model is also stabilized to its origin equilibrium state when using the same FCGs whose selection is based on the LST of fractional-order systems with time delay. Moreover, these numerical results point out that the state variables of the controlled FOS equations are controlled to the origin state 
faster than their time-delay counterpart does when using the same linear controllers.

According to the abovementioned discussion, this work helps us to understand, quantify, and predict the complex dynamics arising from the proposed satellite system. A future work may be devoted to studying the physical interpretation and bifurcation analysis of the FOS model with time delay.

\section{Data Availability}

The data used to support the findings of the study are available from the corresponding author upon reasonable request.

\section{Conflicts of Interest}

The authors declare that they have no conflicts of interest.

\section{Acknowledgments}

The authors specially thank the Deanship of Scientific Research, Majmaah University for his generous support for the accomplishment of this task under project no. R-2021-94.

\section{References}

[1] A. E. Matouk, Advanced Applications of Fractional Differential Operators to Science and Technology, IGI Global, Hershey, PA, USA, 2020.

[2] A. E. Matouk, "Stability conditions, hyperchaos and control in a novel fractional order hyperchaotic system," Physics Letters A, vol. 373, no. 25, pp. 2166-2173, 2009.

[3] A. E. Matouk, "Complex dynamics in susceptible-infected models for COVID-19 with multi-drug resistance," Chaos, Solitons \& Fractals, vol. 140, p. 110257, 2020.

[4] A. E. Matouk and I. Khan, "Complex dynamics and control of a novel physical model using nonlocal fractional differential operator with singular kernel," Journal of Advanced Research, vol. 24, pp. 463-474, 2020.

[5] E. Ahmed and A. E. Matouk, "Complex dynamics of some models of antimicrobial resistance on complex networks," Mathematical Methods in the Applied Sciences, vol. 44, no. 2, pp. 1896-1912, 2021.

[6] A. Zenkour and A. Ahmed, "Fractional thermoelasticity model of a 2D problem of mode-I crack in a fibre-reinforced thermal environment," Journal of Applied and Computational Mechanics, vol. 5, no. 2, pp. 269-280, 2019.

[7] A. Ahmed and H. Ahmad, "A modified thermoelastic fractional heat conduction model with a single lag and two different fractional orders," Journal of Applied and Computational Mechanics, vol. 7, no. 3, pp. 1676-1686, 2020.

[8] M. B. Riaz, I. Siddique, S. T. Saeed, and A. Atangana, "MHD Oldroyd-B fluid with slip condition in view of local and nonlocal kernels," Journal of Applied and Computational Mechanics, vol. 7, no. 1, pp. 116-127, 2020.

[9] M. B. Riaz, S. T. Saeed, and D. Baleanu, "Role of magnetic field on the dynamical analysis of second grade fluid: an optimal solution subject to non-integer differentiable operators," Journal of Applied and Computational Mechanics, vol. 7, no. 1, pp. 54-68, 2021.
[10] M. Caputo, "Linear models of dissipation whose $Q$ is almost frequency independent-II," Geophysical Journal International, vol. 13, no. 5, pp. 529-539, 1967.

[11] I. Podlubny, Fractional Differential Equations, Academic Press, Cambridge, MA, USA, 1999.

[12] M. Caputo and M. Fabrizio, "A new definition of fractional derivative without singular kernel," Progress in Fractional Differentiation and Applications, vol. 1, no. 2, pp. 73-85, 2015.

[13] E. N. Lorenz, "Deterministic nonperiodic flow," Journal of the Atmospheric Sciences, vol. 20, no. 2, pp. 130-141, 1963.

[14] H. N. Agiza and A. E. Matouk, "Adaptive synchronization of Chua's circuits with fully unknown parameters," Chaos, Solitons \& Fractals, vol. 28, no. 1, pp. 219-227, 2006.

[15] T. Matsumoto, "A chaotic attractor from Chua's circuit," IEEE Transactions on Circuits and Systems, vol. 31, pp. 1055-1058, 1984.

[16] G. Chen and T. Ueta, "Yet another chaotic attractor," International Journal of Bifurcation and Chaos, vol. 9, no. 7, pp. 1465-1466, 1999.

[17] A. E. Matouk, "Chaos, feedback control and synchronization of a fractional-order modified autonomous van der polduffing circuit," Communications in Nonlinear Science and Numerical Simulation, vol. 16, no. 2, pp. 975-986, 2011.

[18] S. Kumar, A. E. Matouk, H. Chaudhary, and S. Kant, "Control and synchronization of fractional-order chaotic satellite systems using feedback and adaptive control techniques," International Journal of Adaptive Control and Signal Processing, vol. 35, no. 4, pp. 484-497, 2021.

[19] A. E. Matouk, "Chaos and bifurcations in a discretized fractional model of quasi-periodic plasma perturbations," International Journal of Nonlinear Sciences and Numerical Simulation, 2021.

[20] A. E. Matouk, "A novel fractional-order system: chaos, hyperchaos and applications to linear control," Journal of Applied and Computational Mechanics, vol. 7, no. 2, pp. 701-714, 2021.

[21] J. Hale and H. Kocak, Dynamics and Bifurcations, SpringerVerlag, New York, NY, USA, 1991.

[22] C. Li, X. Yu, W. Yu, T. Huang, and Z.-W. Liu, "Distributed event-triggered scheme for economic dispatch in smart grids," IEEE Transactions on Industrial Informatics, vol. 12, no. 5, pp. 1775-1785, 2015.

[23] C. Li, X. Yu, T. Huang, and X. He, "Distributed optimal consensus over resource allocation network and its application to dynamical economic dispatch," IEEE Transactions on Neural Networks and Learning Systems, vol. 29, no. 6, pp. 2407-2418, 2018.

[24] L. Zhang and J. L. G. Chen, "Extension of Lyapunov second method by fractional calculus," Pure and Applied Mathematics, vol. 21, pp. 291-294, 2005.

[25] Y. Wang and T. Li, "Stability analysis of fractional-order nonlinear systems with delay," Mathematical Problems in Engineering, vol. 2014, Article ID 301235, 8 pages, 2014.

[26] D. Baleanu, A. N. Ranjbar, S. J. Sadati et al., "Lyapunovkrasovskii stability theorem for fractional systems with delay," Romanian Journal of Physics, vol. 56, no. 5-6, pp. 636-643, 2011.

[27] Y. Wen, X.-F. Zhou, Z. Zhang, and S. Liu, "Lyapunov method for nonlinear fractional differential systems with delay," Nonlinear Dynamics, vol. 82, no. 1-2, pp. 1015-1025, 2015.

[28] J.-B. Hu, G.-P. Lu, S.-B. Zhang, and L.-D. Zhao, "Lyapunov stability theorem about fractional system without and with delay," Communications in Nonlinear Science and Numerical Simulation, vol. 20, no. 3, pp. 905-913, 2015. 
[29] M. J. Sidi, Spacecraft Dynamics and Control a Practical Engineering Approach, Cambridge University Press, Cambridge, UK, 1997.

[30] R. W. Zhang, Satellite Orbit and Attitude Dynamics and Control, Beihang University Press, Beijing, China, 1998, in Chinese.

[31] W. MacKunis, K. Dupree, S. Bhasin, and W. E. Dixon, "Adaptive neural network satellite attitude control in the presence of inertia and CMG actuator uncertainties," in Proceedings of the 2008 American Control Conference, Seattle, WA, USA, June 2008.

[32] L.-L. Show, J.-C. Juang, and Y.-W. Jan, "An LMI-based nonlinear attitude control approach," IEEE Transactions on Control Systems Technology, vol. 11, no. 1, pp. 73-83, 2003.

[33] S. Djaouida, "Synchronization of a perturbed satellite attitude motion," International Journal of Mechanical and Mechatronics Engineering, vol. 8, no. 4, pp. 734-738, 2014.

[34] A. P. M. Tsui and A. J. Jones, "The control of higher dimensional chaos: comparative results for the chaotic satellite attitude control problem," Physica D: Nonlinear Phenomena, vol. 135, pp. 41-62, 2000.

[35] J. Kuang, S. H. Tan, and A. Y. T. Leung, "Chaotic attitude motion of satellites under small perturbation torques," Journal of Sound and Vibration, vol. 235, no. 2, pp. 175-200, 2000.

[36] J. Kuang, S. Tan, K. Arichandran, and A. Y. T. Leung, "Chaotic dynamics of an asymmetrical gyrostat," International Journal of Non-linear Mechanics, vol. 36, no. 8, pp. 1213-1233, 2001.

[37] L. Y. Kong, F. Q. Zhoul, and I. Zou, "The control of chaotic attitude motion of a perturbed spacecraft," in Proceedings of the 25th Chinese Control Conference, Harbin, China, August 2006.

[38] T. Liu and J. Zhao, Dynamics of Spacecraft, Harbin Institute of Technology Press, Harbin, China, 2003, in Chinese.

[39] A. Khan and S. Kumar, "Measure of chaos and adaptive synchronization of chaotic satellite systems," International Journal of Dynamics and Control, vol. 7, no. 2, pp. 536-546, 2019.

[40] A. Khan and S. Kumar, "T-S fuzzy modeling and predictive control and synchronization of chaotic satellite systems," International Journal of Modelling and Simulation, vol. 39, no. 3, pp. 203-213, 2019.

[41] A. Khan and S. Kumar, "Analysis and time-delay synchronisation of chaotic satellite systems," Pramana, vol. 91, no. 4, p. 49, 2018.

[42] K. Diethelm, N. J. Ford, and A. D. Freed, "A predictor-corrector approach for the numerical solution of fractional differential equations," Nonlinear Dynamics, vol. 29, pp. 3-22, 2002.

[43] K. Diethelm, "An algorithm for the numerical solution of differential equations of fractional order," Electronic Transactions on Numerical Analysis, vol. 5, pp. 1-6, 1997.

[44] S. Bhalekar and V. Daftardar-Gejji, "A predictor-corrector scheme for solving nonlinear delay differential equations of fractional order," Journal of Fractional Calculus and Applications, vol. 1, no. 5, pp. 1-9, 2011.

[45] N. Aguila-Camacho, M. A. Durate-Mermoud, and J. A. Gallegos, "Lyapunov functions for fractional order systems," Communications in Nonlinear Science and $\mathrm{Nu}$ merical Simulation, vol. 19, no. 9, pp. 2951-2957, 2014.

[46] S. Liang, R. Wu, and L. Chen, "Adaptive pinning synchronization in fractional-order uncertain complex dynamical networks with delay," Physica A: Statistical Mechanics and Its Applications, vol. 444, pp. 49-62, 2016.
[47] F.-F. Wang, D.-Y. Chen, X.-G. Zhang, and Y. Wu, "The existence and uniqueness theorem of the solution to a class of nonlinear fractional order system with time delay," Applied Mathematics Letters, vol. 53, pp. 45-51, 2016.

[48] A. Al-Khedhairi, A. E. Matouk, and S. S. Askar, "Computations of synchronisation conditions in some fractional-order chaotic and hyperchaotic systems," Pramana, vol. 92, p. 11, 2019. 